\title{
Etudes ethnobotaniques des plantes utilisées dans le traitement des parasitoses digestives des petits ruminants (ovins) dans le Sud-Ouest du Niger
}

\author{
Abdoul Razak Issa GARBA ${ }^{1,2^{*}}$, Hassane ADAKAL ${ }^{2}$, Tougiani ABASSE ${ }^{3}$, \\ Koffi KOUDOUVO ${ }^{4}$, Saley KARIM ${ }^{2}$, Adamou AKOURKI ${ }^{2}$, Messanvi GBEASSOR ${ }^{4}$ et \\ Saadou MAHAMANE ${ }^{2}$ \\ ${ }^{1}$ Laboratoire Central d'élevage ; Ministère d'Agriculture et de l'élevage ; BP: 485 Niamey. \\ ${ }^{2}$ Université Dan Dicko Dankoulodo de Maradi, BP: 456 Maradi. \\ ${ }^{3}$ Institut National de la Recherche Agronomique du Niger (INRAN) ; BP : 429 Niamey. \\ ${ }^{4}$ Laboratoire de Physiologie/ Pharmacologie, Département de Physiologie animale / Pharmacologie, Faculté \\ des Sciences, Centre de Recherche et de Formation sur les Plantes Médicinales (CERFOPLAM), Université de \\ Lomé ; BP: 1515 Lomé. \\ *Auteur correspondant; E-mail: razakissa2001@yahoo.fr; Tel: 00227915057 93/0022797941938
}

\section{REMERCIEMENTS}

Les auteurs adressent leurs remerciements au Projet de Productivité Agricole en Afrique de l'Ouest $(P P A A O)$ du Niger pour avoir mis à leur disposition des moyens financiers pour la réalisation de la présente étude.

\section{RESUME}

La présente étude vise à mieux comprendre les méthodes traditionnelles de soins utilisés par les éleveurs et agropasteurs, et d'autre part, à recenser les plantes exploitées pour le traitement des parasites gastrointestinaux dans le Sud-Ouest du Niger. Les éleveurs sont confrontés à des pertes énormes liées aux problèmes de santé du bétail. Ces derniers utilisent alors une multitude de méthodes traditionnelles pour traiter plusieurs affections dont les parasitoses digestives. L'objectif de l'étude est d'inventorier les méthodes traditionnelles de soins utilisées par les éleveurs pour traiter les parasitoses digestives dans les régions Sud-Ouest (Tillabéry, Dosso et Niamey) du Niger. Pour ce faire, une enquête d'interviews semi-structurées auprès de 801 éleveurs et agropasteurs a été conduite dans trois régions du Niger. Les résultats montrent que les éleveurs (100\%) utilisent la médecine traditionnelle pour soigner les animaux malades. Douze pour cent des éleveurs/agropasteurs font appelle en second lieu aux vétérinaires et $15 \%$ aux tradipraticiens. La moyenne d'âge de ces derniers se situe entre 42 et 50 ans et la majorité $(92,60 \%)$ est analphabète. Les remèdes traditionnels proposés pour lutter contre les parasites digestifs des petits ruminants (ovins) dans ces régions sont essentiellement à base de plantes locales composées de 33 espèces appartenant à 21 familles botaniques. Les modes de préparation et d'administration de ces plantes sont variés et diffèrent d'un éleveur à un autre. Ceci permettra de proposer le traitement le plus efficace sans aucun risque de toxicité pour l'animal en vue de leurs utilisations ultérieures dans un cadre sécurisé pour les éleveurs et agropasteurs.

(C) 2019 International Formulae Group. All rights reserved

Mots clés: Pharmacopée vétérinaire, parasites digestifs, tradipraticien, Niger. 


\title{
Ethnobotany studies of plants used in the treatment of digestive parasitizes of small ruminants (sheep) in South-Western Niger
}

\begin{abstract}
Traditional methods used by pastoralists in treating gastrointestinal parasites of small ruminants in Southwestern Niger were studied while the different plants used for this purpose are assessed. Animal health problems are currently faced by farmers in rural areas. To alleviate such health problems, traditional methods are used in treating various infections, among which the gastrointestinal parasites. To assess that, 801 farmers were interviewed through a survey conducted in three south-western regions of Niger (Tillabéry, Dosso and Niamey). Results showed that, $100 \%$ of farmers interviewed relied on traditional medicine for animal health issues, $12 \%$ called on veterinary services while $15 \%$ on traditional healers. Most of those farmers $(92.6 \%)$ are illiterate and aged 42 to 50years old. 33 species of local plants belonging to 21 botanic families are mainly used for this purpose. The processing and route of administration of those plants differ from one farmer to the other. With that, the most effective way of treatment, having less toxicity for both farmers and animals, will be sought out for future usage.
\end{abstract}

(C) 2019 International Formulae Group. All rights reserved

Keywords: veterinary pharmacopoeia, Digestive parasites, traditional healers, Niger.

\section{INTRODUCTION}

L'élevage est une activité économique essentielle en Afrique de l'Ouest (CEDEAO, 2011). L'élevage des ovins est consacré à la satisfaction des besoins d'alimentation quotidienne chez les nomades à travers les productions de lait et de viande (Rhissa, 2010). Au Niger, l'élevage tient une place importante dans l'économie nationale. Il est pratiqué par près de $87 \%$ de la population active soit en tant qu'activité principale, soit comme activité secondaire après l'agriculture (Rhissa, 2010). De plus en plus une attention particulière est mise sur les petits ruminants à cause de leur importance numérique et de leur rôle dans les exploitations agricoles. Mais de nos jours les éleveurs sont confrontés à d'énormes contraintes telles que la faible productivité des animaux, l'insuffisance des fourrages liés aux déficits pluviométriques et surtout les problèmes de santé qui occasionnent des pertes physique du bétail. Pour Tamssar (2006), l'élevage est confronté aux problèmes de maladies infectieuses, nutritionnelles et parasitaires.
La santé du bétail est donc perturbée par plusieurs pathologies. Thiaucourt et al. (2011) ont montré que la pathologie constitue l'un des obstacles les plus importants à l'amélioration de la productivité des troupeaux de petits ruminants en Afrique. La santé animale est un élément indispensable pour l'amélioration des performances du cheptel (Zaibet et al., 2008). Pour Cabaret (2004), le parasitisme est considéré comme un élément essentiel de la gestion d'un troupeau de ruminants. L'infestation des petits ruminants par des parasites gastro-intestinaux constitue un problème sanitaire et économique très important (AGRIDEA, 2007).

Les taux de prévalence aux strongles digestifs sont élevés et varient entre 70 à $100 \%$ (Belem et al., 2000). Les pertes zootechniques globales engendrées par ces strongyloses gastro-intestinales peuvent représenter jusqu'au tiers de la productivité (Mensah et al., 2006), d'où la nécessité de mener une lutte rationnelle contre cette pathologie.

Cela suscite une résistance aux antihelminthiques (Foteini, 2011). Comme 
solution, les plantes médicinales constituent des ressources précieuses pour la grande majorité des populations rurales en Afrique (Dibong et al., 2011). Plus de $80 \%$ de la population ouest africaine ont recours à la médicine traditionnelle en cas de maladie (WHO, 2002). La pratique courante de la médecine traditionnelle est sans aucun doute attribuable à son efficacité, son accessibilité et son coût abordable et aux facteurs tels que la culture, l'histoire et les convictions personnelles (Elisabeth, 2008). Ces recettes représentent une source non négligeable de nouveaux médicaments surtout qu'elles présentent moins d'effets secondaires (Moroh et al., 2008 ; Kokora et al., 2013). Plusieurs espèces de plantes ont été identifiées comme des plantes médicinales utilisées pour le traitement de diverses infections (Sopkon et Ouinsavi, 2002 ; Biecke, 2004). L'inventaire exhaustif de toutes ces recettes qui restent jusque-là à notre connaissance non disponibles s'avèrent indispensables afin de ressortir les meilleurs remèdes pour le traitement des parasitoses digestives chez les petits ruminants. Cette investigation permettra de proposer aux acteurs de la filière, des recettes efficientes de traitement adaptées au contexte socioéconomique préservant l'écologie. La présente étude vise à recenser par enquête ethnobotanique les remèdes traditionnels prescrits par les éleveurs et agropasteurs au Sud-Ouest du Niger pour lutter contre les parasitoses digestives des petits ruminants (ovins).

\section{MATERIEL ET METHODES \\ Milieu d'étude}

L'étude s'est déroulée d'août à septembre 2015, au Niger. Le climat est caractérisé par trois saisons : chaude (de mars à mai), pluvieuse (de juin à septembre) et froide (d'octobre à février). La région Tillabéry est traversée par le fleuve Niger. C'est un plateau à environ $250 \mathrm{~m}$ d'altitude coupés par des cours d'eau temporaires ou permanents. Le climat est de type sahélien, chaud et relativement humide avec $540 \mathrm{~mm}$ de précipitations à Niamey. Elle ne reçoit cependant pas une goutte de pluie pendant plus de 4 mois par an, et les précipitations sont irrégulières. Quant à la région de Dosso, elle possède un climat désertique avec une température moyenne de $29,5{ }^{\circ} \mathrm{C}$ et les précipitations sont en moyenne de $635,3 \mathrm{~mm}$. Le climat à Niamey est de type sahélien (semi-aride) (INS, 2015). La végétation est constituée de savanes arborées arbustives, et aussi des steppes épineux et d'acacias (Figure 1). Le choix de ces trois sites s'est fait à cause de la diversité ethnique des éleveurs et agropasteurs pratiquant l'élevage.

\section{Méthode}

Les informations ont été collectées par une interview semi structurée suivant les méthodes décrites par Maphosa et al. (2010) et Victor (2010). Les villages enquêtés sont choisis en fonction de la concentration des petits ruminants (ovins). L'enquête a été réalisée auprès de 801 éleveurs et agropasteurs (dont $47,1 \%$ à Tillabéry, $35,3 \%$ à Dosso et $17,6 \%$ à Niamey) dans les trois régions selon un échantillonnage aléatoire proportionnel. Ces éleveurs ont été choisis sur la base du type d'élevage (ovins) et de la disponibilité à participer à l'enquête. Il s'agit d'obtenir des informations précises sur les pratiques d'élevage, les soins de santé animale, les recettes pour le traitement des parasitoses digestives des ovins et l'origine de ces recettes. A l'issue de cette étape, chaque enquêté participait à l'identification d'une personne ressource bien connue présent sur le terroir et à laquelle il fait recours en cas de besoins pour les soins traditionnels. Ces personnes ressources ont été aussi questionnées. Les informations recueillies au niveau de tous les enquêtés ont porté sur leur profil socio-économique, leur connaissance des parasitoses des petits ruminants et l'identification des plantes médicinales 
utilisées pour le traitement de ces pathologies (identification, description, techniques de préparation, modes d'administration et mode de conservation des remèdes). La reconnaissance botanique de toutes les plantes proposées par les éleveurs et agropasteurs s'est faite avec l'aide des éleveurs/agropasteurs et des spécialistes de l'INRAN (Institut National de la Recherche Agronomique du Niger) sur le terrain et la confirmation avec PROTA $4 \mathrm{U}$. Le questionnaire d'enquête a été testé et validé au préalable par une pré-enquêtes au près des éleveurs avant la phase d'enquête proprement dite. Dans le cadre du présent travail nous nous sommes limités aux résultats issus des entretiens avec les éleveurs et agropasteurs des différentes zones d'intervention.

\section{Analyse statistique}

Les données collectées ont été dépouillées manuellement, codifiées et saisies avec le logiciel EpiData ${ }^{\circledR}$ 3.1. avant d'être exporté vers le logiciel Excel. Elles ont été soumises enfin à un calcul de moyennes et de fréquences avec le logiciel SPSS (version 17.0) et $\mathrm{R}^{{ }^{\circ}}$ 3.1.2.

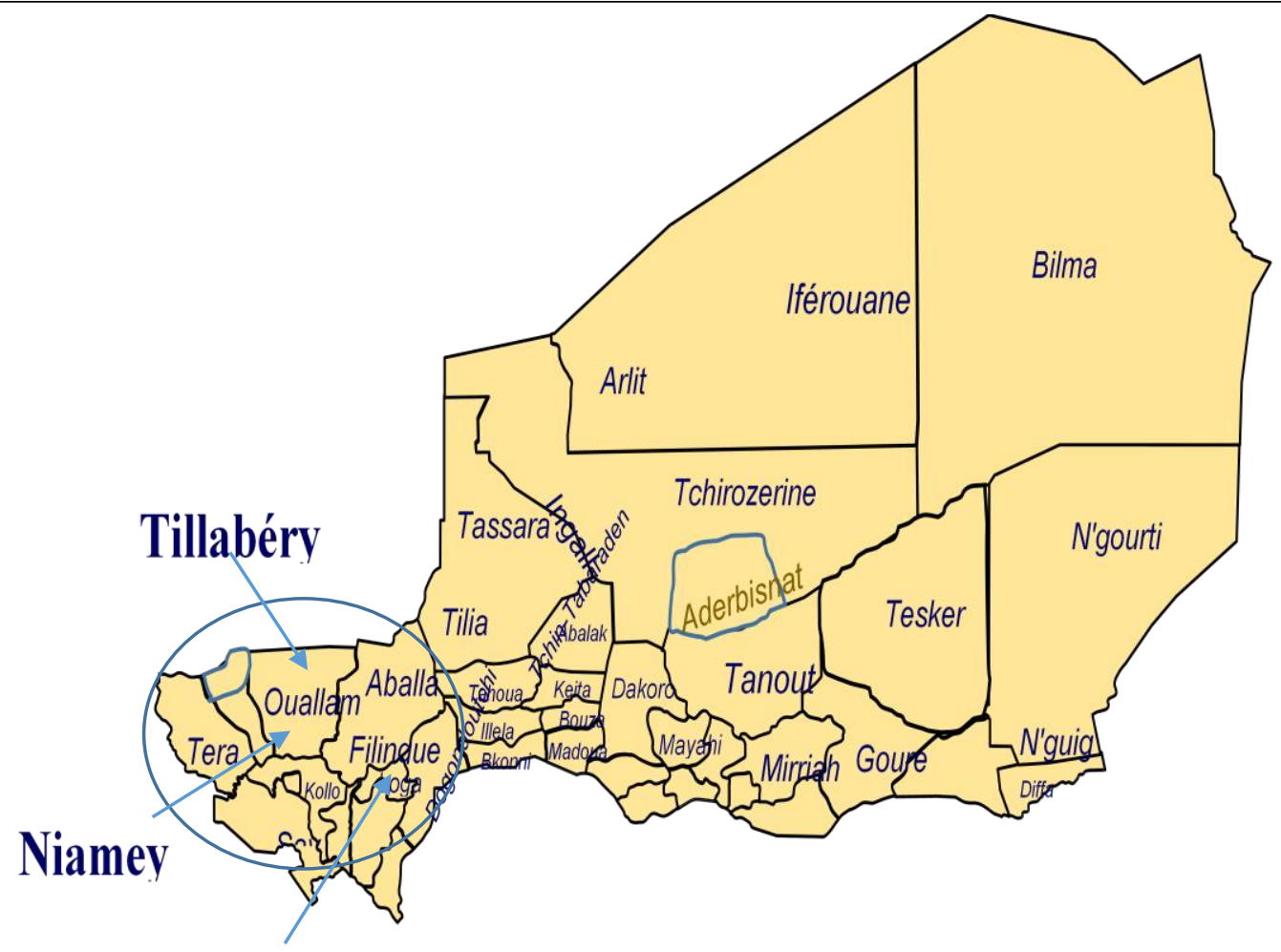

Dosso

Figure 1: Zone d'étude. 


\section{RESULTATS}

Répartition des enquêtés en fonction de l'âge et du niveau d'instruction, du groupe ethniques et du sexe

Huit cent un éleveurs dont 4 femmes ont été identifiés par la population comme des personnes recommandées dans les trois régions enquêtés (Figure 2). Tous ont accepté de se soumettre aux questionnaires. Il s'agit principalement des groupes ethniques peulh majoritaire qui sont représenté à 88,80\%, haoussa, gourmantché, Kourteye, Touareg et Sonrhaï (Figure 3). Ces derniers sont soit des éleveurs purs, soit des agro-pasteurs ou agriculteurs. Un virgule quarante-neuf pour cent sont alphabétisés et $5,86 \%$ ont suivi des enseignements à l'école coranique (Figure 4). L'âge des personnes interrogées varie entre 25 et 85 ans avec une moyenne de 50,42 $\pm 12,96$ ans.

\section{Recours à la médecine traditionnelle vétérinaire}

Les résultats obtenus montrent que l'ensemble des éleveurs enquêtés font recours à la médecine traditionnelle pour soigner leurs animaux malades. Néanmoins $12 \%$ font appel à un vétérinaire en seconde lieu et $15 \%$ aux tradipraticiens, après avoir traité eux même leurs animaux traditionnellement, tandis que les $72 \%$ font l'automédication (Figure 10). Pour les enquêtés, les raisons qui expliquent ces observations sont entre autre l'absence ou l'indisponibilité de l'agent vétérinaire au bon moment pour soigner les animaux malades, l'efficacité reconnue aux remèdes traditionnels, l'inefficacité de certains traitements modernes et enfin le respect de la tradition.

\section{Connaissance des symptômes du parasitisme des petits ruminants (Ovins) par les enquêtés}

En ce qui concerne les principaux facteurs de l'apparition des parasites digestifs chez les ovins, $75 \%$ des enquêtés affirment que ce sont les pâturages souillés qui favorise ces infections. Mais pour certain éleveurs (24\%) ce sont les eaux des mares souillées et des fleuves qui sont à l'origine de l'infection parasitaire. Tous ces éléments sont reconnus favorables à l'apparition et/ou à l'accentuation de la prévalence de l'infestation des petits ruminants. Seulement $1 \%$ n'ont aucune idée de ces facteurs, Comme le montre la Figure 7. Le diagnostic de la maladie se fonde sur les symptômes cliniques observés sur les animaux malades. Les principaux signes cliniques que décrivent les éleveurs/agropasteurs sont par ordre décroissant l'anorexie $(51,8 \%)$, les poils piqués $(25,5 \%)$, la présence de vers dans les fèces $(17,5 \%)$, et la diarrhée $(5,2 \%)$ (Figure 6). Ces signes sont observables en toute saison, avec une prédominance en saison des pluies.

\section{Remèdes traditionnels contre le parasitisme digestif des petits ruminants}

Trente-cinq (35) remèdes traditionnels contre le parasitisme digestif des petits ruminants ont été prescrits par les éleveurs et agropasteurs comme étant efficaces contre les parasitoses digestives des petits ruminants (Ovins). Le Tableau 1 présente ces remèdes. Du point de vue analytique, ils sont composés essentiellement de végétaux et sont généralement préparés extemporanément au moment de l'utilisation. Les recettes à une plante sont représentées à 92,8\%. Des ingrédients tel que le sel est parfois ajoutés. De façon générale, 33 espèces végétales appartenant à 21 familles botaniques ont été identifiées. Khaya senegalensis a été la plante la plus citée par les enquêtés $(23,72 \%)$, suivie de Boscia senegalensis (12,48\%), Tephrosia purpurea (9,86\%), Maerua crassifoilia $(8,11 \%)$, Adansonia digitata $(4,11 \%)$ et de Anogeissus leiocarpa (3,99\%) (Tableau 1). Les principales parties des plantes utilisées dans la préparation de ces remèdes sont les feuilles $(45,81 \%)$, les écorces $(31,71 \%)$, les tiges feuillées $(14,48 \%)$, les racines $(4,88 \%)$ et les graines $(3,12 \%)$ (Figure 5 ). Ces parties sont récoltées le jour, notamment au cours de la matinée, parfois très tôt. Certaines plantes comme striga hermonthica sont récoltées uniquement en saison des pluies. Les instruments utilisés pour la récolte sont constitués souvent de pioches, de coupecoupes et de couteaux. Les formes galéniques des remèdes les plus utilisées par les éleveurs et agropasteurs sont la macération, la décoction et l'infusion. Les opérations de macération et de décoction sont effectuées dans des canaris en argile ou dans des calebasses. A l'issue de ces préparations, la solution à administrer à l'animal est filtrée avec un tissu propre avant d'être utilisée. A chaque répétition du traitement, la même procédure de préparation est renouvelée. L'administration des remèdes se fait principalement par la voie orale. La durée des traitements varie d'un (1) à trois (3) jours en fonction de la recette. La posologie des recettes est également variable et dépend de la forme galénique. 
Tableau 1 : Recettes traditionnelles préconisées par les éleveurs/agropasteurs dans le traitement des parasitoses digestives des petits ruminants (Ovins).

\begin{tabular}{|c|c|c|c|c|c|}
\hline Plantes ou produits utilisés & Espèces & Familles & Organes & $\begin{array}{l}\text { Mode de } \\
\text { préparation }\end{array}$ & Voies d'administration \\
\hline \multicolumn{6}{|l|}{ Langues locales ou nature du produit } \\
\hline Koo nya (Z), Kuka(H), Bokki (P) & Adansonia digitata & Bombacaceae & $\mathrm{Fe}$ & Macération & $\begin{array}{l}\text { Per os } 2 \text { fois/jour pendant } 2 \text { jours dans } \approx 1 \text { litre } \\
\text { d'eau }\end{array}$ \\
\hline Gonga (Z), Maréké(H), Kodioli(P) & Anogeissus leiocarpa & & $\mathrm{Fe} / \mathrm{Ec}$ & Infusion & Per os 3 fois/jour pendant 2 jours. \\
\hline Déli-nya(Z), Tarammya(H), Dohki(P) & Combretum glutinosum & Combretaceae & $\mathrm{Fe}$ & Macération & Per os 2 fois/jour (matin et soir) pendant 2 jours \\
\hline Kubu(Z), Géza(H), Gumumi (P) & Combretum micranthum & & Ec & Décoction & Per os 2 fois/jour pendant 3 jours. \\
\hline Sabara (Z), Sabara (H), Gélokhi (P) & Guiera senegalensis & & $\mathrm{Fe}$ & Macération & Per os 1fois/Jour. \\
\hline Mai Gudaigi(H), Fayraré(P) & Spermacoce stachydea & Rubiaceae & $\mathrm{R}$ & Macération & Per os en une seule fois (Matin). \\
\hline Kabey (Z), Giayia (H), Koli (P) & Mitragyna inermis & & Ec & Macération & Per os 2 fois/jour \\
\hline Shinkiliga (Z), Zuré $(\mathrm{H})$, Tirei $(\mathrm{P})$ & Boscia salicifolia & & $\mathrm{Tf}$ & Macération & Per os 2 fois/jour (matin et soir) pendant 3 jours \\
\hline Anza (Z), Anza (H), Gigilé (P) & Boscia senegalensis & Capparaceae & $\mathrm{Fe}$ & Macération & Per os 1 fois/jour (matin) pendant 2 jours \\
\hline Bagey (Z), Bagey (H), Bagahy (P) & Cadaba farinosa & & $\mathrm{Tf}$ & Macération & Per os 2 fois/jour (matin et soir) pendant 3 jours \\
\hline Hasu Bio (Z), Jiga (H), Hasuhi(P) & Maerua crassifolia & & $\mathrm{Fe}$ & Macération & Per os 2 fois/jour pendant 2 jours \\
\hline Sééfoy (Z), Dukki (H), N'gannky (P) & Celtis integrifolia & Celtidaceae & $\mathrm{R}$ & Macération & Per os 1 fois/jour pendant 2 jours. \\
\hline Bagarbey (Z), Rawaya (H), Yarudi (P) & Cochlospermum planchonii & Cochlospermaceae & $\mathrm{Fe}$ & Macération & Per os, pilées et mises dans l'eau d'abreuvement \\
\hline Gudjya'r awaki $(\mathrm{H})$ & Crotalaria rotusa & & $\mathrm{Fe}$ & Macération & Per os ad libitum jusqu'à la guérison dans l'eau. \\
\hline Kosey (Z),Kalgo (H), Barki (P) & Pilostigma reticulatum & & Ec & Macération & Per os 2 fois/jour (matin et soir) pendant 2 jours \\
\hline
\end{tabular}


A. R. I. GARBA et al. / Int. J. Biol. Chem. Sci. 13(3): 1534-1546, 2019

\begin{tabular}{|c|c|c|c|c|c|}
\hline Masa (Z), Masa (H), Masahy $(\mathrm{P})$ & Tephrosia purpurea & Fabaceae & $\mathrm{Tf}$ & Macération & Per os une seule prise \\
\hline Zam Turi (Z), Kiriya (H), Kohy (P) & Prosopis africana & & $\mathrm{Fe} / \mathrm{Ec}$ & Décoction & Per os en une seule prise \\
\hline Bani (Z), Marjee $(\mathrm{H})$ & Acacia nilotica & & $\mathrm{Fe}$ & Macération & Per os 2 fois/jour pendant 3 jours. \\
\hline Sisan (Z), Malga (H), Malgahi (P) & Cassia sieberiana & & $\mathrm{R}$ & Décoction & Per os 2 fois/jour pendant 3 jours. \\
\hline Kokoyé (Z), Gao(H), Chaski (P) & Acacia albida & & Ec & Macération & Per os 2 fois/jour jusqu'à guérison. \\
\hline Tondi Bonhawéy (Z), Koriba (H) & Croton zambesicus & Euphorbiaceae & $\mathrm{G}$ & Macération & Per os plus du sel 2 fois /Jours. \\
\hline Kullum (Z), Komaya (H), Bululé (P) & Eragrostis tremula & Poaceae & $\mathrm{R}$ & Macération & Per os 1 fois/Jours \\
\hline Farey (Z), Mad'atchi (H), Kahi (P) & Khaya senegalensis & Meliaceae & Ec & Macération & Per os 2 fois/Jours le matin et soir. \\
\hline $\begin{array}{l}\text { Gangi-Dunou }(\mathrm{Z}) \text {, Taramnyia }(\mathrm{H}) \text {, } \\
\text { Jirlahi(P) }\end{array}$ & Kigelia africana & Bignoniaceae & $\mathrm{Ec}$ & Décoction & Per os en une seule prise. \\
\hline Tamarza (Z), Farun dawiya (H), Faruhi (P) & Lannea acida & Anacardiaceae & $\mathrm{Fe} / \mathrm{Ec}$ & Décoction & Per os en une seule prise. \\
\hline Lallé (Z), Lallé (H) & Lawsonia inermis & Lythraceae & $\mathrm{Tf}$ & Macération & Per os 2 fois /Jours \\
\hline Hanamm (Z), Yadya $(\mathrm{H})$, Yadyahul (P) & Leptadania hastata & Asclepiadaceae & $\mathrm{Fe}$ & Macération & Per os 2 fois /Jours. \\
\hline Hiro (Z), Talaekia (H), Katiak (P) & Salvadora persica & Salvadoraceae & $\mathrm{Fe}$ & Décoction & Per os en une seule prise. \\
\hline Diney (Z),Dania (H), Hédi (P) & Sclerocarya bierrea & Anacardiaceae & $\mathrm{Fe} / \mathrm{Ec}$ & Macération & Per os 2 fois/Jours. \\
\hline Zata (Z), Sanya (H), Alali (P) & $\begin{array}{l}\text { Securidaca } \\
\text { longepedunculata }\end{array}$ & Polygalaceae & $\mathrm{Tf}$ & Macération & Per os 2 fois/Jours. \\
\hline Malli (Z), Gawguyé(H), Mahé (P) & Striga hermonthica & Scrophulariaceae & $\mathrm{Tf}$ & Macération & Per os une seule prise. \\
\hline Daré $(\mathrm{Z})$, Magaria $(\mathrm{H})$, Djabhi $(\mathrm{P})$ & Ziziphus mauritiana & Rhamnaceae & $\mathrm{Ec}$ & Décoction & Per os 2 fois/jour pendant 3jours. \\
\hline Garbay (Z), Adua (H), Tanni & Balanites aegyptiaca & Zygophyllaceae & $\mathrm{Ec}$ & Décoction & $\begin{array}{l}\text { per os } 2 \text { fois/jour, pendant } 2 \text { jours dans } 1 / 2 \text { litre } \\
\text { d'eau }\end{array}$ \\
\hline
\end{tabular}

Z: Langue Zarma H : Langue Haoussa P : Langue Peulh $\quad \mathrm{Fe}$ : Feuille, Tf : Tige feuillée, Ec : Ecorce, R : Racine, G : Graine 


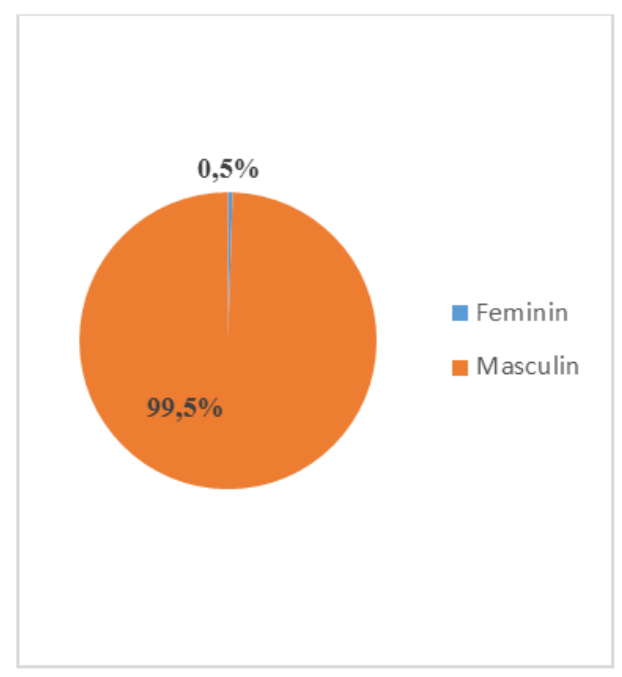

Figure 2 : Répartition de la population en fonction du sexe.

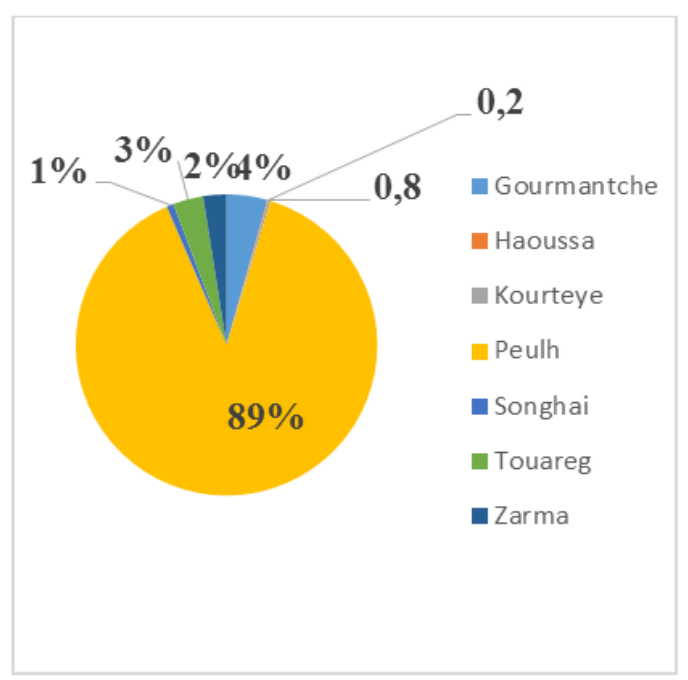

Figure 3: Répartition de la population en fonction des ethnies.

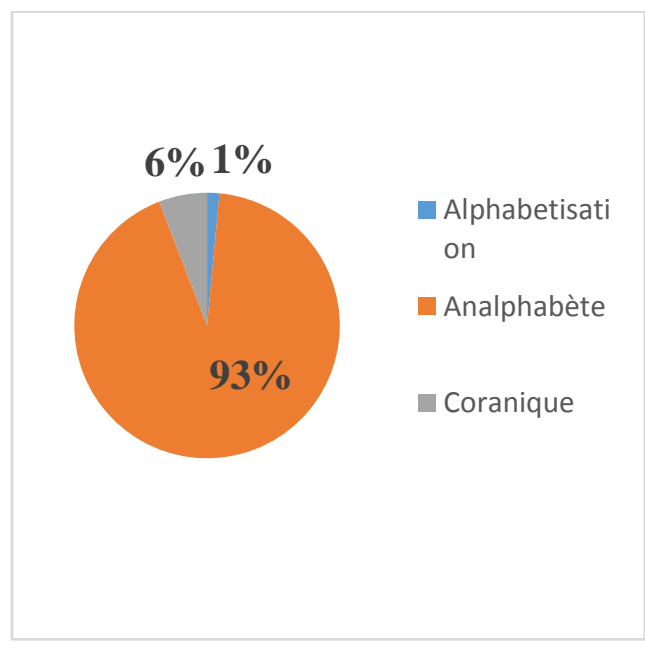

Figure 4 : Répartition de la population en fonction du niveau d'étude

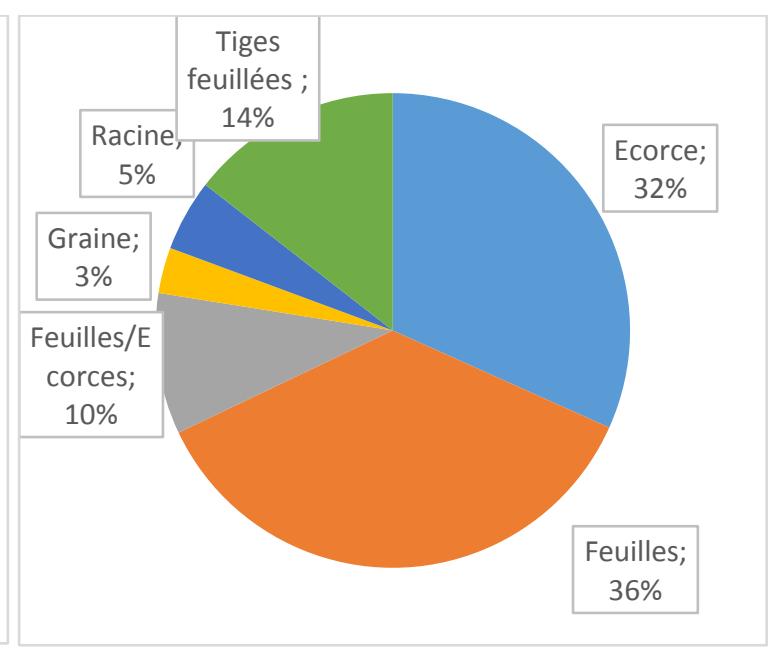

Figure 5: Parties de plantes utilisées par les éleveurs/agropasteurs 


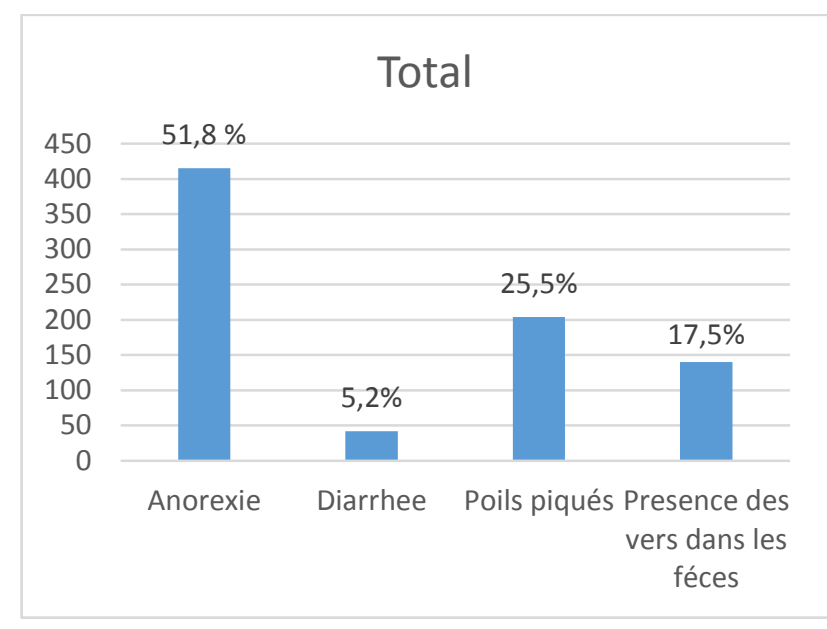

Figure 6: Principaux signes décrites par les éleveurs/agropasteurs.

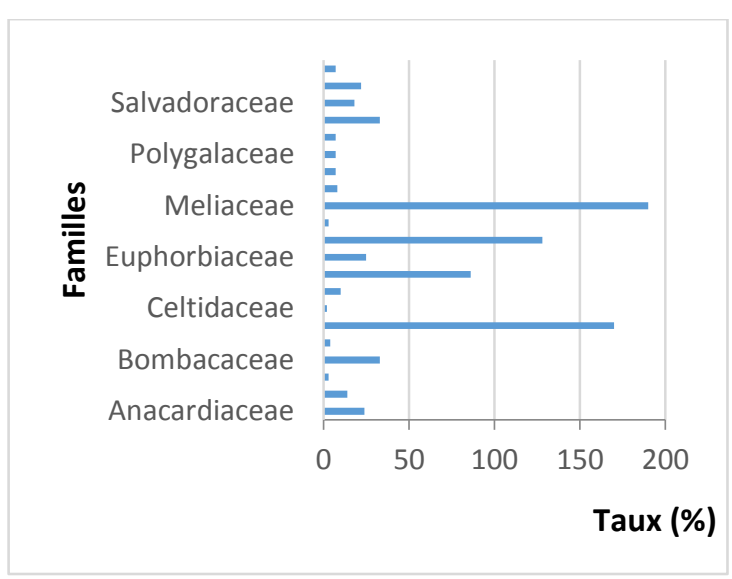

Figure 8: Répartition des espèces déclarées parmi les familles botaniques.

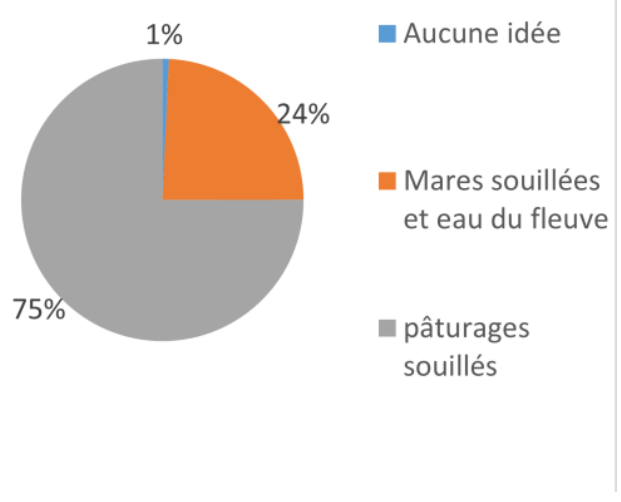

Figure 7 : Facteurs de risques décrits par les éleveurs/agropasteurs.

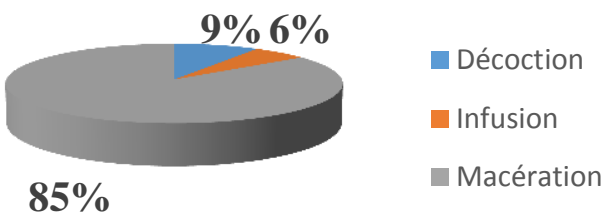

Figure 9 : Modes de préparation utilisés par les éleveurs/agropasteurs.

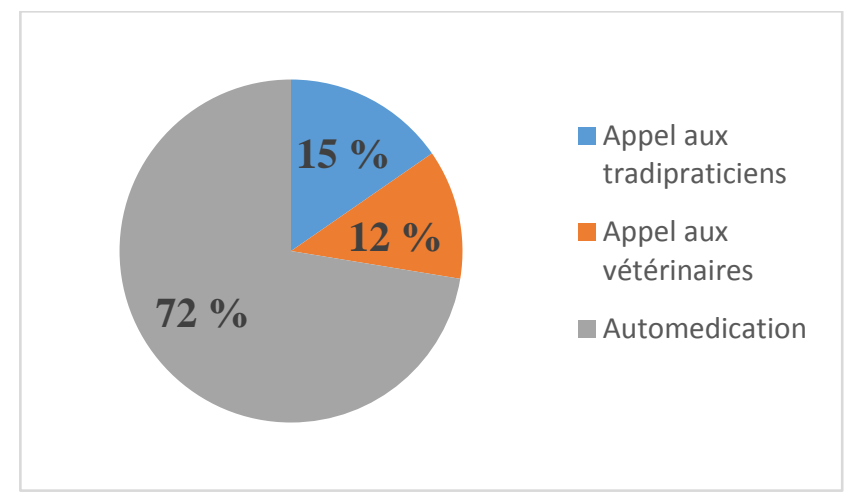

Figure 10: Méthodes de traitement utilisées par les éleveurs /agropasteurs. 


\section{DISCUSSION}

Cette étude a permis de ressortir les différentes recettes utilisées par les éleveurs/agropasteurs dans le traitement des parasitoses digestives des ovins. D'une façon générale, trente-trois (33) espèces appartenant à vingt et une (21) familles botaniques ont été inventoriées. Les familles les plus prédominantes sont Meliaceae, Capparaceae, Fabaceae, Combretaceae et Bombacaceae. Ces résultats sont en adéquation avec les résultats de recherches de Kaboré et al. (2007). Ces chercheurs ont montré que les familles des plantes représentées sont aussi les Combretaceae, Bombacaceae, Meliaceae etc. Mais ces résultats diffèrent de ceux trouvés par Benarba (2016) qui a révélé que les familles des plantes les plus prédominants étaient Lamiacées, Asteraceae, Apiaceae et Fabaceae. Pour la formulation des formes galéniques des plantes, les éleveurs utilisent toutes les parties de la plante. De toutes ces parties, les feuilles sont les plus utilisées dans le traitement des parasitoses digestives des ovins. Des observations similaires ont été faites par Kaboré et al. (2007) et Benarba (2016). Ces chercheurs ont montré respectivement que $56,2 \%$ des éleveurs au Burkina Faso utilisaient les feuilles dans le traitement des parasitoses digestives des petits ruminants alors qu'au Sud-Ouest de l'Algérie seulement 36\% des guérisseurs locaux l'utilisaient. Concernant le mode de préparation, il ressort que la macération (85\%) est le principal mode de préparation des espèces médicinales. Ces résultats ont été confirmés par Kabore et al. (2007),qui ont montré que les principales modes de préparation de ces plantes au Burkina Faso sont la macération et la décoction. Par ailleurs, il y a une prédominance de l'administration par voie orale des différentes plantes médicinales dans le sud-ouest du Niger. Cette observation est en accord avec les résultats d'études de Koné et al. (2005), Benarba et al. (2014), Chermat et al. (2015), qui ont notifié que la voie orale est la plus dominante dans l'administration des différentes plantes médicinales .Signalons que la notion des doses des médicaments traditionnels n'est pas respectée chez les enquêtés. Les éleveurs et agropasteurs font recours à la médecine traditionnelle pour les soins de santé des animaux dans les différentes zones d'étude. Certain éleveurs $(12,23 \%)$ font appelle en second lieu à la médecine moderne. Pour Agaie et al. (2007) ce résultat peut expliquer par le fait que les interventions ethno-vétérinaires sont souvent la première ligne de défense contre les problèmes de santé. La médecine ethnovétérinaire diffère non seulement d'une région à l'autre mais aussi au sein des communautés (Mathias, 2001; Tamboura, 2006). Cette médication traditionnelle fondée sur les savoirs endogènes du terroir et appliquée à la gestion de la bonne santé des animaux est faite essentiellement à base de plantes, comme l'ont mentionné Kaboré et al (2007). Elle est dispensée par des personnes relativement âgées et investies d'une certaine confiance de la part des éleveurs et agro-pasteurs du milieu dans lequel ils vivent. La majorité des éleveurs font encore confiance en ces méthodes et savoirs endogènes comme l'ont évoqué Tamboura et al. (1998), Kaboré et al. (2007) au Burkina Faso, Barry et al. (2001) en Guinée Conakry, ainsi que HounzangbéAdoté (2005) au Bénin. En effet, les raisons qui poussent les éleveurs/agropasteurs à recourir à la médecine vétérinaire traditionnelle sont nombreuses et variées. Au nombre de ces raisons la disponibilité des médicaments de bonne qualité et l'efficacité après usage de ces derniers. Ceci peut être due par l'utilisation anarchique des médicaments par les éleveurs/agropasteurs et viens alors confirmer nos observations sur le terrain (nonrespect de la dose des médicaments vétérinaires et leur utilisation abusive). Parmi toutes ces raisons, le motif le plus fondamental semble être le manque d'agent vétérinaire dans les villages et leur disponibilité au moment opportun. De plus certains éleveurs sont encore très attachés aux coutumes de leurs villages. Cette étude vient confirmer l'existence des traitements des maladies animales par la médecine traditionnelle en générale et celui des parasitoses digestives des petits ruminants (ovins) en particulier. De plus, l'oralité reste également le moyen essentiel de conservation et de transmission de connaissances des plantes médicinales au sein de la famille. Ces 
résultats confirment bien les observations rapportées par Barry et al. (2001) qui ont travaillé au Fouta Djallon (Guinée Conakry). Aussi, la transmission du savoir et le savoirfaire se fait avec une grande importance au sein des familles par $48,4 \%$ des enquêtés. Ce qui explique la conservation de l'information de génération en génération, bien que les jeunes ne soient pas assez coopératifs. Les jeunes (20 à 30 ans) ne sont pas assez représentés dans cette étude. Cela s'explique qu'à ce niveau l'étude réalisée en saison des pluies s'est intéressée à ce moment rien qu'aux personnes ressources et comme nous avons travaillé pendant la saison des pluies, les jeunes sont souvent occupés aux travaux de champs et ne sont donc pas disponibles. Le nombre réduit des femmes dans l'échantillonnage peut s'expliquer par le fait que dans les zones d'étude, elles sont rarement propriétaires d'animaux, ou bien elles sont représentées par leurs maris. Les résultats de l'enquête montrent que les éleveurs/agropasteurs ont une idée claire des symptômes des parasitoses digestives des petits ruminants. Ces informations sont semblables à celles faites par Belem et al. (2005b) qui a noté une phase d'hypermétabolisme parasitaire de juin à octobre avec les conclusions scientifiques dans le même contexte écologique que cette étude. Aryam et al., (2008) a évoqué également ces mêmes idées dans ces travaux de recherches effectuées au Niger dans la région de Tahoua sur l'apparition des maladies parasitaires. Les enquêtés considèrent les symptômes décrits comme synonymes de mauvaise santé chez l'animal à travers les modifications de son comportement normal ou de son état général. La survenue de ces signes fait l'objet d'une intervention à but thérapeutique pour éviter la mortalité du jeune sujet et/ou la baisse des performances zootechniques au niveau des adultes. Par ailleurs, les remèdes inventoriés sont composés essentiellement des végétaux rencontrés dans les différentes zones d'étude. Ce résultat est en accord avec l'étude réalisée par Hounzangbé-Adoté (2005), Githiori et al. (2005), Kaboré et al. (2007), et Koné et al. (2005). Ces remèdes sont préparés au moment de l'emploi ou très peu de temps avant l'utilisation. Les plantes à effet anthelminthique citées par les éleveurs/agropasteurs dans l'étude sont en accord avec les observations faites dans la sous-région Ouest- Africaine où certaines plantes identifiées ont déjà été testées scientifiquement. Telles que: Anogeissus leiocarpa testé par Koné et al. (2005) en Côte d'Ivoire; Ademola et al. (2004) au Nigéria; Guiera senegalensis testé par Suleiman et al. (2014) au Nigeria. En effet, face à tous ces facteurs contraignants, la prise en compte du savoir de la médecine traditionnelle africaine peut être une option intéressante à promouvoir pour assurer les soins aux animaux malades (McGAW et ELOFF, 2008).

\section{Conclusion}

En sommes, au lieu de faire recours aux produits pharmaceutiques à coût très élevé dans le traitement des parasitoses digestives chez les ovins, il serait très intéressant de pousser les investigations sur ces différentes recettes. Ceci permettra de proposer le traitement le plus efficace sans aucun risque de toxicité pour l'animal en vue de leurs utilisations ultérieures dans un cadre sécurisé pour les éleveurs et agropasteurs.

\section{CONFLIT D'INTERETS}

Les auteurs déclarent qu'il n'existe aucun conflit d'intérêts relatif à cet article.

\section{CONTRIBUTIONS DES AUTEURS}

ARIG a élaboré le sujet, et a conduit la recherche, la collecte des données sur le terrain, la saisie des données collectées pour analyse statistique et la rédaction du manuscrit ainsi que la réalisation de l'analyse statistique des données collectées sur le terrain avec; HA et $\mathrm{TA}$ a conduit la recherche et a contribué à l'écriture du manuscrit; KK, SK et AK participé à l'élaboration du manuscrit, $\mathrm{MB}$ et SM participé à l'élaboration du sujet, la conduite de la recherche et la contribution à l'écriture du manuscrit.

\section{REMERCIEMENTS}

Nous remercions les autorités locales pour leur soutien. Nous sommes aussi très reconnaissants à l'égard des éleveurs et agropasteurs pour leur disponibilité. 


\section{REFERENCES}

Ademola IO, Faghemi BO, Idowu SO, 2004. Evaluation of the anthelmintic activity of Khaya senegalensis extract against gastrointestinal nematodes of sheep: in vitro and in vivo studies. Veterinary Parasitology, 122: 151-164.

Agaie BM, Onyeyili PA, Muhammad BY, Ladan MJ. 2007. Acute toxicity effects of the aqueous leaf extract of Anogeissus leiocarpus in rats. African Journal of Biotechnology, 6(7): 886-889.

AGRIDEA (Association Suisse pour le Developpement de l'Agriculture et de l'Espace Rural). 2007. Ovins, caprins Parasites gastro-intestinaux. Bio., 13(5): 11-14.

Arya m S, Salla A, Ilhidji el HA, Bangana I, 2008. Maladies Parasitaires Animales, Réponses Thérapeutiques De L'ethnopharmacopée Vétérinaire De La Zone Pastorale De Tahoua / Niger. Médecine Traditionnelle et Pharmacopée, 15 : 36 - 40.

Barry MS, Oularé K, Diallo A, Camara Y, Camara M, Baldé MA. 2001. La medicine traditionnelle au Fouta Djallon: République de Guinée. Revue Méd. Pharm. Afr., 15: 95- 102.

Belem AMG, Kaboré A, Bessin R. 2005a. Gastrointestinal helminths of sheep in the central, eastern and northern parts of Burkina Faso. Bull Anim. Hlth. Prod. Afr., 53: 13-23.

Belem AMG, Nikiéma L, Sawadogo L, Dorchies Ph. 2000. Parasites gastrointestinaux des moutons et risques d'infestation parasitaire des pâturages en saison pluvieuse dans la région centrale du Burkina Faso. Rev. Méd. Vét., 151: 437-442.

Benarba B, Meddah B, Tir Touil A. 2014. Response of bone resorption markers to Aristolochia longa intake by Algerian breast cancer postmenopausal women. Adv Pharmacol Sci., 820589.

Benarba B. 2016. Medicinal plants used by traditional healers from South-West Algeria: An ethnobotanical study. Journal of Intercultural Ethnopharmacology, 10: 0814115725.
Biecke B. 2004. Etnobotanishe studie van geneeskrachtige planten in Manigri en Igbére, Benin. Universiteit Gent., Bioingenieurin hetland, En Bos Beheer, 420p.

Cabaret J. 2004. Parasitisme helminthique en élevage biologique ovin: réalités et moyens de contrôle. INRA Production Animale, 17 (2) 145-154.

Chermat S, Gharzouli R. 2015.Ethnobotanical study of medicinal flora in the North East of Algeria, An empirical knowledge in Djebel Zdimm (Setif). J Mater Sci Eng., 5: 50-9. 26.

Dibong SD, Mpondo Mpondo E, Ngoye A, Betti JL, 2011. Ethnobotanique et phtytomédecine des plantes médicinales vendues sur les marchés de Douala, Cameroun. Journal of Applied Biosciences, 37 : 2496-2507.

CEDEAO (Communauté économique des Etats de l'Afrique de l'Ouest). 2011. Les moyens sont disponibles pour aller de l'avant.

http://www.essor.ml/societe/article/eleva ge-dans-I-espace-cedeao-Ies.

Elisabeth B. 2008. Les changements climatiques : vulnérabilité, impacts et adaptation dans le monde de la médecine traditionnelle au Burkina Faso. Vertigo la revue électronique en sciences de l'environnement, 8(1). URL : http://vertigo.revues.org/index1467.html (consulté le 28 mars 2009).

Foteini M. 2011. Propriétés anthelminthiques du sainfoin (Onobrychis viciifoliae) : Analyse des facteurs de variations et du rôle des composés phénoliques impliqués 26.

Githiori JB, Hôglund J, Waller PJ. 2005. Ethnoveterinary plant preparations as livestock dewormers: practices, popular beliefs, pitfalls and prospects for the future. Anim. Health Res. Rev., 6(1): 91103.

Hounzangbé-Adoté SM. 2005. Propriétés anthelminthiques de 4 plantes tropicales testées in vitro et in vivo sur les nématodes gastro-intestinaux chez les petits ruminants Djallonké. Thèse de Doctorat, Université d'Abomey-Calavi, Benin, $240 \mathrm{p}$. 
INS (Institut National de la Statistique). 2015. du Niger.

Kaboré A, Tamboura HH, Belem AMG, Traoré A. 2007. Traitements ethnovétérinaires des parasitoses digestives des petits ruminants dans le plateau central du Burkina Faso. Int. J. Biol. Chem. Sci., 1(3): 297-304.

Kokora PA, Ackah JAAB, Nanga ZY, Kra MAK, Loukou GY, Coulibaly A, Djaman J. 2013. Antibacterial activity of ethanolic and aqueous extracts of four medicinal plants on the in vitro growth of escherichia coli and staphylococcus aureus. Journal of Drug Delivery and Therapeutics, 3: 113-116.

Koné MW, Kamanzi AK, Traoré D, Betschart B. 2005. Anthelmintic activity of medicinal plants used in Northern Côte d'Ivoire against intestinal helminthiasis. Pharmaceutical Biology, 43(1): 72-78.

Maphosa V, Masika PJ. 2010. Ethnoveterinary uses of medicinal plants: a survey of plants used in the ethnoveterinary control of gastrointestinal parasites of goats in the Eastern Cape Province, South Africa. Pharm Biol., 48: 697-702.

Mc Gaw LJ, ELOFF JN. 2008. Ethnoveterinary use of Southern African plants and scientific evaluation of their medicinal properties. $J$. Ethnopharmacol., 119: 559-574.

Mensah GA, Sobakin LJ, Koudande D, Pomalegni CB, Kpera GN. 2006. Inventaire préliminaire des plantes médicinales utilisées pour traiter les aulacodes d'élevages malades et pour la prophylaxie sanitaire dans les aulacodicultures installés au Sud-Bénin. Bulletin de la Recherche Agronomique du Bénin, (54).

Moroh JLA, Bahi C, Dje K, Loukou YG, Guede- guina F. 2008. Étude de l'activité antibactérienne de l'extrait acétatique (EAC) de Morinda morindoides (Baker) milne-redheat (rubiaceae) sur la croissance in-vitro des souches
d'Escherichia coli. Bulletin de la Société Royale des Sciences de Liège, 77: 44- 61. Rhissa Z. 2010 : Revue du secteur d'élevage au Niger. 115p.

Sokpon N, Ouinsavi C. 2002. Utilisation du Khaya senegalensis en médicine traditionnelle au Bénin ; Revue de Médecine et de Pharmacopée Africaine, $16: 9-14$.

Suleiman MM, Mamman M, Sidiama A, Ighoja EJ, Tauheed M, Talba AM. 2014. Evaluation of anthelmintic activity of Nigerian ethnoveterinary plants; Cassia occidentalis Guiera senegalensis, Veterinary World, EISSN: 2231-0916.

Tamssar MN. 2006. Parasitisme helminthique gastro-intestinal des moutons abattus aux abattoirs de dakar. Thèse de Doctorat Vétérinaire, E.I.S.M.V, Université Cheikh Anta Diop de Dakar. 67p.

Tamboura HH. 2006. Activité biologique des extraits aqueux de Holarrhena floribunda (G. Don) Durand \& Schinz (Apocynaceae) : Etude des effets de type hormonale mâle chez le rat. Doctorat d'Etat en Sciences Biologiques Appliquées, Université de Ouagadougou. 176 pages + annexes.

Thiaucourt F, Fikre J, Mebratu G, Guerin C, Antonio DM. 2011. Quelles peuvent être les priorités de recherche dans le domaine de la pathologie des petits ruminants en Afrique. http://www.fao.org/wairdocs/ilri/x5520b/ x5520bOq.htm.

Victor O. 2010. Activité antihelminthique de la poudre d'écorce de racine de Vitex thomasii de Wild (Verbenaceae) sur Haemonchus contortus chez la chèvre. $258 \mathrm{p}$

WHO. 2002. Traditional Medicines Strategy 2002-2005. WHO/EDM/TRM/2002.1: Geneva; Switzerland.

Zaibet L, Hammani S, Jabbar M. 2008. Durabilités des systèmes d'élevage des petits ruminants en Tunisie: Une approche de Santé animale et marketing. ILRI. 139p. 